\title{
Effects of tolvaptan on renal function in chronic kidney disease patients with volume overload
}

This article was published in the following Dove Press journal: International Journal of Nephrology and Renovascular Disease

\author{
Shunji Suzuki ${ }^{1,2}$ \\ Norio Hanafusa ${ }^{3}$ \\ Kenji Kubota' \\ Ken Tsuchiya ${ }^{3}$ \\ Kosaku Nitta ${ }^{2}$ \\ 'Department of Nephrology, \\ Kawaguchi Saiseikai Hospital, \\ Kawaguchi, Japan; ${ }^{2}$ Department of \\ Medicine, Kidney Center, Tokyo \\ Women's Medical University, \\ Tokyo, Japan; ${ }^{3}$ Department of Blood \\ Purification, Kidney Center, Tokyo \\ Women's Medical University, Tokyo, \\ Japan
}

Correspondence: Kosaku Nitta Department of Medicine, Kidney Center, Tokyo Women's Medical University, 8-I Kawada-cho, Shinjuku-ku, Tokyo 162-8666, Japan

Tel $+813335381 \mathrm{II}$

$\mathrm{Fax}+8 I 333560293$

Email knitta@twmu.ac.jp
Background: Fluid overload in chronic kidney disease (CKD) is generally controlled by diuretics, with potentially harmful effects on renal function. The efficacy of tolvaptan, a vasopressin V2-receptor antagonist and aquaretic, has not been evaluated for fluid control in CKD with reduced renal function.

Methods: Each patient from a group of $24 \mathrm{CKD}$ patients on tolvaptan $15 \mathrm{mg} / \mathrm{d}$ plus conventional diuretics ( $\mathrm{T}$ group) was matched by age and sex with a patient from a group of $24 \mathrm{CKD}$ patients on conventional nonaquaretic diuretics alone not associated to tolvaptan other than tolvaptan (C group). Changes in renal function were compared between the groups for 1 year. Results: There were no significant differences in blood pressure, hemoglobin levels, cardiac function, urine specific gravity, and urinary sodium concentration between the 2 groups at the beginning of the follow-up period and 1 year after. The estimated glomerular filtration rate (eGFR) by the formula developed by Japanese Society of Nephrology (in $\mathrm{mL} / \mathrm{min} / 1.73 \mathrm{~m}^{2}$ ) decreased: C group (from 28.3 \pm 13.6 to $23.0 \pm 12.3, p=0.09$ ), T group (from $22.7 \pm 12.4$ to $19.4 \pm 12.2, p=0.18$ ), but both did not reach significance. A $50 \%$ reduction in eGFR was observed in 4 patients in the $\mathrm{C}$ group and 1 in the $\mathrm{T}$ group $(p<0.05)$. A subgroup analysis performed on the patients with stage 3-4 CKD demonstrated a significant reduction in eGFR in the $\mathrm{C}$ group $(\mathrm{n}=17, p=0.04)$, but not in T group $(\mathrm{n}=17, p=0.07)$.

Conclusion: These results suggest that tolvaptan may have less effects on CKD progression among stage 3-4 CKD patients who are on conventional diuretics.

Keywords: tolvaptan, eGFR preservation, observational study, diuretics

\section{Background}

Treatment of chronic kidney disease (CKD) includes early intervention to prevent further deterioration of renal function and cardiovascular complications. From this perspective, early identification of CKD and appropriate management can reduce the risk of end-stage kidney disease and of renal replacement therapy (RRT), and can improve survival by mitigating cardiovascular risk and slowing CKD progression.

Loop diuretics and thiazides are generally used to control fluid and sodium overload in CKD. Edema is frequently observed in CKD and is well controlled by these diuretics. However, a prospective observational study on predialysis patients suggested that the association of high doses of these diuretics contributed to progression to RRT. ${ }^{1}$

Tolvaptan is a newly developed vasopressin receptor antagonist that induces diuresis and has been found efficacious in treating heart failure ${ }^{2}$ and preventing the growth of renal cysts in autosomal dominant polycystic kidney disease (ADPKD) 
patients. A short-term study showed that tolvaptan was also effective in controlling volume status in CKD with ${ }^{3}$ or without ${ }^{4}$ heart failure. Tolvaptan can also be used for fluid management in patients with diabetic nephropathy. ${ }^{5}$ However, the long-term effects on renal function in CKD patients remain unknown. Therefore, we investigated whether tolvaptan associated with conventional diuretics affected renal function over 1 year in CKD patients with fluid overload.

\section{Methods}

\section{Study design}

This was a retrospective observational study. According to a by-law on the approval by the Japanese Ministry of Health and Welfare, tolvaptan cannot be used alone and must be combined with conventional diuretics. Therefore, we investigated the effect of tolvaptan when associated with conventional diuretics in the patients previously diagnosed as having heart failure.

\section{Study population}

The potential population for this study consisted of 200 outpatients with CKD treated from 2011 to 2016 at Kawaguch Saiseikai Hospital. From this population, we identified 24 patients who were taking tolvaptan $(15 \mathrm{mg})$ with other diuretics (T group). From the same population of 200, we selected 24 patients who were matched with each patient in the $\mathrm{T}$ group by age, sex, primary diagnosis other than tolvaptan, and serum creatinine (C group). Subjects with active infection, decompensated liver disease, acute kidney injury, and autoimmune disorders were excluded. Moreover, participants who were pregnant and/or had malignancies, pacemakers, and implanted metal devices were also excluded from the study. The patient's demographics were recorded from the hospital database. Fluid overload was defined by the presence of leg edema, increased cardiothoracic ratio on chest X-ray, and weight gain.

\section{Drug exposure}

The doses of diuretics before and after the study period are shown in Table 1. Doses of furosemide were adjusted and modified based on the level of edema. Data on medications were collected from medical records.

\section{Evaluation}

Data on blood chemistry, blood pressure, and echocardiogram from dates nearest to the start of tolvaptan were chosen as the baseline data, and those nearest to 1 year later were used for follow-up data. Estimated glomerular filtration rate (eGFR) was calculated from serum creatinine values using an equation for the Japanese developed by the Japanese Society of Nephrology (eGFR $\left[\mathrm{mL} / \mathrm{min} / 1.73 \mathrm{~m}^{2}\right]=194 \times \mathrm{sCr}^{-1.094} \times$ $\mathrm{Age}^{-0.287}$ for men and $=194 \times \mathrm{sCr}^{-1.094} \times \mathrm{Age}^{-0.287} \times 0.793$ for women). ${ }^{6}$ Plasma brain-type B natriuretic peptide was

Table I Baseline characteristics of study subjects

\begin{tabular}{|c|c|c|c|c|c|}
\hline & \multicolumn{2}{|c|}{ Control group $(n=24)$} & \multicolumn{2}{|c|}{ Tolvaptan group $(n=24)$} & \multirow[t]{2}{*}{$p$-value } \\
\hline & Mean & SD & Mean & SD & \\
\hline Age (years) & 70.4 & 16 & 76.5 & 11.2 & 0.14 \\
\hline Sex (male, \%) & 80 & & 80 & & 1.0 \\
\hline Primary disease (diabetes, \%) & 38 & & 41 & & 0.77 \\
\hline Creatinine $(\mathrm{mg} / \mathrm{dL})$ & 2.2 & 0.84 & 2.4 & 0.086 & 0.41 \\
\hline BNP (log Pg/mL) & 2.1 & 0.5 & 2.1 & 0.15 & 0.74 \\
\hline Urine specific gravity & 1.01 & 0.005 & 1.009 & 0.005 & 0.11 \\
\hline Ejection fraction $(\%)$ & 65 & 12 & 65 & 12 & 0.8 \\
\hline Right ventricular pressure $(\mathrm{mmHg})$ & 32.5 & 14.2 & 30 & 8.0 & 0.9 \\
\hline Hemoglobin $(g / d L)$ & 11.8 & 2.3 & 10.8 & 1.7 & 0.09 \\
\hline Sodium $(\mathrm{mEq} / \mathrm{L})$ & 139 & 3.3 & 139 & 3.5 & 0.65 \\
\hline Systolic BP (mmHg) & $14 \mid$ & 18.7 & 140 & 14.4 & 0.88 \\
\hline Diastolic BP (mmHg) & 69 & 15.2 & 63 & 9.9 & 0.1 \\
\hline ARB & $71 \%$ & & $63 \%$ & & 0.54 \\
\hline Statin & $33 \%$ & & $8 \%$ & & 0.03 \\
\hline Charcoal & $29 \%$ & & $62 \%$ & & $0.02^{\mathrm{a}}$ \\
\hline EPA & $4 \%$ & & $13 \%$ & & 0.3 \\
\hline Ezetimibe & $0 \%$ & & $8 \%$ & & 0.15 \\
\hline Uric acid (mg/dL) & 6.8 & 1.4 & 6.7 & 1.4 & 0.74 \\
\hline
\end{tabular}

Note: ${ }^{a} p<0.05$ vs control group.

Abbreviations: ARB, angiotensin receptor blocker; BNP, brain natriuretic peptide; BP, blood pressure; EPA, eicosapentaenoic acid; n, number of patients; SD, standard deviation. 
measured at the time of entry as previously described. ${ }^{7}$ Echocardiography was performed to evaluate the ejection fraction and right ventricular pressure as previously described. ${ }^{8}$

Edema status was evaluated in semiquantitative manner. In the medical records, the degree of the edema found in each patient was described in 3 categories, namely, none, trace, and present. Thus, we transformed these statuses into a score of 0,1 , and 2 , respectively.

\section{Statistical analysis}

The demographic and clinical characteristics of the patients were summarized as mean and standard deviation for continuous variables, and numbers and proportions for categorical variables. Categorical and continuous variables were compared using the $\chi^{2}$ test and the 2-sample $t$-test, respectively. Variables that could be assumed to follow normal distribution were tested using $F$-test. Percentile differences were tested using Mann-Whitney $U$-test. A 2 -sided $p$-value of $<0.05$ was considered as an indicator of statistical significance. All the analyses were performed using Excel EZR.

\section{Ethical issues}

This study was conducted in line with the guidelines in the Declaration of Hesinki. All the procedures of the study were approved by the Research Ethics Board of Kawaguchi Saiseikai Hospital (No. 29-5). Written informed consent was obtained from all of the subjects.

\section{Results}

Baseline demographics and clinical characteristics of study subjects are shown in Table 1 . There were no significant differences between groups in age, sex distribution, serum creatinine; there were no significant differences in B-type natriuretic peptide, right ventricular pressure, blood pressure, serum sodium concentration, uric acid, urine specific gravity, hemoglobin, diabetes as the causes of CKD $(\mathrm{C} ; 38 \%, \mathrm{~T} ; 41 \%)$ body weight $(C ; 64 \pm 15, T ; 60 \pm 11 \mathrm{~kg})$, albumin $(C ; 3.5 \pm 0.7$, $\mathrm{T} ; 3.4 \pm 0.7 \mathrm{mg} / \mathrm{dL})$, cardiothoracic ratio $(\mathrm{C} ; 51.8 \pm 5.0 \%$, T; $51.0 \pm 5.7 \%)$, pretibial edema $(\mathrm{C} ; 1.2 \pm 0.9, \mathrm{~T} ; 1.3 \pm 0.9$ that was expressed [-] as $0,[ \pm]$ as 1 , and [+] as 2), and medications such as angiotensin II receptor blockers, ezetimibe, and eicosapentaenoic acid. Significant differences were only found in use of a statin and charcoal.

All of the 48 CKD patients (24 from the T group and 24 from the $\mathrm{C}$ group) had clinical data recorded that allowed renal function to be assessed for the 1-year observation period. No patient started RRT or was hospitalized. No patient exhibited overt heart failure (New York Heart Association class III and
IV) or liver cirrhosis (Child Pugh class C). One patient had nephrotic syndrome, but the amount of proteinuria did not change. Although doses of furosemide could be modified according to fluid status, there were no significant differences in these doses between $\mathrm{C}$ and $\mathrm{T}$ groups. Other medications including diuretics were not added or changed during the observation period.

Tables 2 and 3 show the results of blood chemical analysis, blood pressure, and echocardiogram findings before and at the end of 1-year of follow-up. None of these parameters changed significantly for each patient during the observation period. Semiquantitative edema status was unchanged from 1.2 \pm 0.9 to $1.1 \pm 1.0$ in $\mathrm{C}$ group and from $1.3 \pm 0.9$ to $1.0 \pm 1.0$ in $\mathrm{T}$ group. Serum sodium concentration and urine specific gravity both in the $\mathrm{C}$ and $\mathrm{T}$ groups did not change during the study period, suggesting that $\mathrm{T}$ group may have a higher water intake. Nonetheless, body weight tended to decrease in T group.

Renal function did not change significantly during the 1 -year observation period in both groups. Mean serum creatinine $(\mathrm{mg} / \mathrm{dL})$ concentration increased from 2.2 to 2.8 in group $\mathrm{C}$ and from 2.2 to 2.4 in group T. The eGFR values $\left(\mathrm{mL} / \mathrm{min} / 1.73 \mathrm{~m}^{2}\right)$ did not statistically decrease in the $\mathrm{C}$ group $(p=0.09)$ and in the $\mathrm{T}$ group $(p=0.18)$. The degree of the decrease was greater in the $\mathrm{C}$ group than in the $\mathrm{T}$ group. Thus, we further analyzed this reduction in eGFR. Percent decreases were not different between the 2 groups. However, a $50 \%$ reduction of eGFR was significantly and more frequently observed in the $\mathrm{C}$ group (4/24 cases) than the $\mathrm{T}$ group (1/24 cases, $U$-test, $p<0.05)$.

We further analyzed the decline in eGFR in a subgroup of patients with stage $3 \mathrm{a}-4 \mathrm{CKD}$, since tolvaptan is known to be ineffective in patients with ADPKD of stage 5 (unpublished observation by Otstuka Pharmaceutical Co., Tokyo, Japan). In this subgroup analysis, the eGFR values $\left(\mathrm{mL} / \mathrm{min} / 1.73 \mathrm{~m}^{2}\right)$ in 17 patients in the $\mathrm{C}$ group decreased significantly during the observation period $(29.0 \pm 9.5,27.7 \pm 10.0,23.6 \pm 9.7$; baseline, 1 month, and 1 year, respectively, $p<0.01$ between baseline and 1 year), while the eGFR values in 17 patients in the T group did not change during this period (26.0 \pm 12.7 , 24.6 $\pm 19.0,22.3 \pm 12.0$; baseline 1 month and 1 year respectively) (Figure 1).

\section{Discussion}

This study demonstrated that tolvaptan may ameliorate the decline in renal function among patients with stage $3 a-4$ CKD. The finding is consistent with those of previous reports on patients with heart failure, cirrhosis, ${ }^{9}$ or nephrotic syndrome. ${ }^{10}$ 
Table 2 Changes in biochemical markers at baseline and I year after the start of study in control group

\begin{tabular}{|c|c|c|c|c|c|}
\hline & \multicolumn{2}{|c|}{ Baseline $(n=24)$} & \multicolumn{2}{|c|}{ After I year $(n=24)$} & \multirow[t]{2}{*}{$p$-value } \\
\hline & Mean & SD & Mean & SD & \\
\hline Hemoglobin (g/dL) & 11.8 & 2.3 & 11.7 & 1.5 & 0.88 \\
\hline Sodium (mEq/L) & 139 & 3.3 & 139 & 2.8 & 0.44 \\
\hline Uric acid (mg/dL) & 6.8 & 1.4 & 6.7 & 1.3 & 0.8 \\
\hline Creatinine (mg/dL) & 2.2 & 0.84 & 2.8 & 1.4 & 0.04 \\
\hline eGFR (mL/min/l.73 m²) & 28.3 & 13.6 & 23 & 12.3 & 0.09 \\
\hline Urine specific gravity & 1.01 & 0.005 & 1.01 & 0.004 & 0.95 \\
\hline Ejection fraction (\%) & 65 & 12 & 64 & 11.4 & 0.8 \\
\hline Right ventricular pressure $(\mathrm{mmHg})$ & 32.5 & 10.2 & 33 & 11.5 & 0.9 \\
\hline BNP $(\log \mathrm{pg} / \mathrm{mL})$ & 2.1 & 0.5 & 1.9 & 0.5 & 0.3 \\
\hline Systolic BP (mmHg) & $|4|$ & 18.7 & 140 & 19.2 & 0.3 \\
\hline Diastolic BP (mmHg) & 69 & 15.2 & 70 & 12.3 & 0.6 \\
\hline Cardiothoracic ratio (\%) & 51.8 & 5 & 52 & 5.7 & 0.6 \\
\hline Albumin (mg/dL) & 3.5 & 0.7 & 3.6 & 0.7 & 0.7 \\
\hline Body weight (kg) & 64.5 & 15 & 65.1 & 16 & 0.5 \\
\hline \multicolumn{6}{|l|}{ Subgroup (CKD 3a-4) n=17 } \\
\hline & \multicolumn{2}{|c|}{ Baseline } & \multicolumn{2}{|c|}{ After I year } & $p$-value \\
\hline Hemoglobin (g/dL) & 12.2 & 2.3 & 12.1 & 1.5 & 0.9 \\
\hline Sodium (mEq/L) & 139 & 3.1 & 139 & 1.8 & 0.8 \\
\hline Creatinine (mg/dL) & 1.9 & 0.6 & 2.5 & 1.3 & $0.02^{\mathrm{a}}$ \\
\hline eGFR $\left(\mathrm{mL} / \mathrm{min} / 1.73 \mathrm{~m}^{2}\right)$ & 29.0 & 9.5 & 23.6 & 9.7 & $0.006^{b}$ \\
\hline \multicolumn{6}{|l|}{ Diuretics (mg/d) } \\
\hline Furosemide & 23.8 & 21.6 & 32.1 & 20.3 & 0.11 \\
\hline Thiazide & 0.21 & 0.4 & 0.33 & 0.4 & 0.68 \\
\hline Spironolactone & 9.38 & 8.3 & 6.25 & 9.9 & 1.0 \\
\hline Tolvaptan & 0 & 0 & 0 & 0 & \\
\hline
\end{tabular}

Note: ${ }^{a} p<0.05,{ }^{b} p<0.01$ vs baseline data.

Abbreviations: BNP, brain natriuretic peptide; BP, blood pressure; CKD, chronic kidney disease; eGFR, estimated glomerular filtration rate; $n$, number of patients; SD, standard deviation.

The effect of tolvaptan on heart failure has been established. ${ }^{10}$ A meta-analysis indicates a benefit of tolvaptan in cardiac function. ${ }^{11}$ The studies in the meta-analysis were short-term studies in which tolvaptan was administered for a week, resulting in additional diuresis and amelioration of volume overload. Heart congestion was significantly reduced without affecting eGFR. Another short-term study involving 1 week of tolvaptan in CKD revealed similar diuresis. ${ }^{12}$ In the present study, the duration of therapy was much longer. Measured cardiac function and fluid status indicators such as blood pressure were not altered. This finding suggests that protection of eGFR by tolvaptan was neither via improvement in cardiac function nor fluid volume control.

Tolvaptan was the first agent approved for the treatment of progressive ADPKD in CKD stage $1-3 .{ }^{13}$ A clinical trail demonstrated the safety and benefit of tolvaptan in the progression of ADPKD. ${ }^{14}$ However, our study excluded the patients with ADPKD. Thus, the benefit of tolvaptan on eGFR in this study may not be associated with the effects seen in the patients with ADPKD. This trial showed that the decline in eGFR after 1-year of follow-up was less in the tolvaptan group than in the placebo control group, although an elevation of transaminase was observed..$^{15}$ This effect of protection against the decline of eGFR by tolvaptan was consistent with the results of the present study. However, the dose of tolvaptan in ADPKD (60 mg or more) is at least 4 times higher than the dose used in this study. This much higher dose may have adverse effects on the liver. In our study, the lower dose was not associated with adverse effects and had similar effects on eGFR, suggesting that tolvaptan is useful in moderate CKD (stage $3 \mathrm{a}-4$ ) but not in advanced CKD (stage 5). Because our study did not include patients with ADPKD, the benefit of tolvaptan on eGFR may have been direct rather than a result of controlling the size of renal cysts.

Thus, the mechanism by which tolvaptan protects renal function from decline in CKD remains speculative. One explanation might be associated with the fact that tolvaptan allows the patients to have greater fluid intake, as patients in the T group would have required more fluid than those in the $\mathrm{C}$ group to maintain normal extracellular fluid volume. 
Table 3 Changes in biochemical markers at baseline and I year after the start of study in tolvaptan group

\begin{tabular}{|c|c|c|c|c|c|}
\hline & \multicolumn{2}{|c|}{ Baseline $(n=24)$} & \multicolumn{2}{|c|}{ After I year $(n=24)$} & \multirow[t]{2}{*}{$p$-value } \\
\hline & Mean & SD & Mean & SD & \\
\hline Hemoglobin (g/dL) & 10.8 & 0.8 & 11.2 & 1.4 & 0.45 \\
\hline Sodium $(\mathrm{mEq} / \mathrm{L})$ & 139 & 0.7 & 138 & 4.1 & 0.44 \\
\hline Uric acid (mg/dL) & 6.9 & 0.7 & 6.7 & 1.8 & 0.8 \\
\hline Creatinine $(\mathrm{mg} / \mathrm{dL})$ & 2.4 & 0.86 & 2.9 & 1.3 & 0.12 \\
\hline eGFR (mL/min/l.73 m²) & 22.7 & 12.4 & 19.4 & 12.2 & 0.18 \\
\hline Urine specific gravity & 1.01 & 0.005 & 1.008 & 0.005 & 0.78 \\
\hline Ejection fraction (\%) & 65 & 12 & 60 & 12.3 & 0.22 \\
\hline Right ventricular pressure $(\mathrm{mmHg})$ & 30 & 8.0 & 35 & 13.0 & 0.18 \\
\hline BNP $(\log \mathrm{pg} / \mathrm{mL})$ & 2.1 & 0.5 & 2.1 & 0.6 & 0.9 \\
\hline Systolic BP (mmHg) & $|4|$ & 18.7 & 137 & 17.0 & 0.9 \\
\hline Diastolic BP $(\mathrm{mmHg})$ & 63 & 9.7 & 64 & 8.9 & 0.14 \\
\hline Cardiothoracic ratio (\%) & 51 & 5.7 & 51 & 6.7 & 0.7 \\
\hline Albumin (mg/dL) & 3.4 & 0.7 & 3.5 & 0.6 & 0.7 \\
\hline Body weight (kg) & 60 & 11 & 58.6 & 11 & 0.45 \\
\hline \multicolumn{6}{|l|}{ Subgroup (CKD G3a-4) n= 17} \\
\hline & \multicolumn{2}{|c|}{ Baseline } & \multicolumn{2}{|c|}{ After I year } & $p$-value \\
\hline Hemoglobin (g/dL) & 11.1 & 1.8 & 11.5 & 1.4 & 0.48 \\
\hline Sodium (mEq/L) & 139 & 3.7 & 139 & 3.8 & 0.7 \\
\hline Creatinine $(\mathrm{mg} / \mathrm{dL})$ & 2.0 & 0.6 & 2.4 & 0.9 & $0.02^{\mathrm{a}}$ \\
\hline eGFR $\left(\mathrm{mL} / \mathrm{min} / \mathrm{l} .73 \mathrm{~m}^{2}\right)$ & 26.0 & 12.7 & 22.3 & 12 & 0.07 \\
\hline \multicolumn{6}{|l|}{ Diuretics (mg/d) } \\
\hline Furosemide & 32.9 & 20.3 & 48.3 & 54.5 & 0.1 \\
\hline Thiazide & 0.29 & 0.4 & 0.22 & 0.4 & 0.7 \\
\hline Spironolactone & 9.38 & 2.9 & 12.5 & 11.8 & 0.63 \\
\hline Tolvaptan & 9.84 & 3.7 & 11.3 & 3.5 & 0.1 \\
\hline
\end{tabular}

Note: ${ }^{a} p<0.05$, vs baseline data.

Abbreviations: BNP, brain natriuretic peptide; BP, blood pressure; CKD, chronic kidney disease; eGFR, estimated glomerular filtration rate; n, number of patients; SD, standard deviation.

eGFR $\left(\mathrm{mL} / \mathrm{min} / 1.73 \mathrm{~m}^{2}\right)$

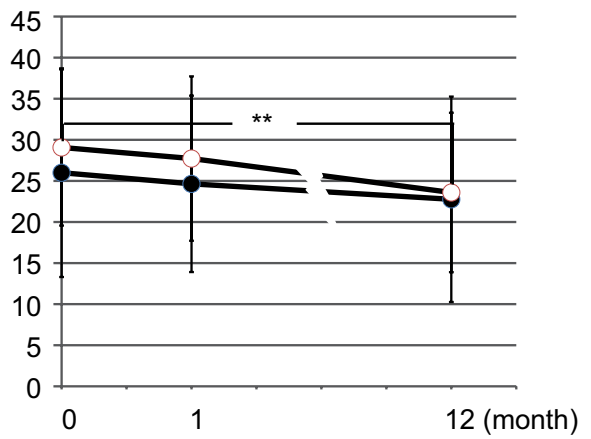

Figure I Changes in eGFR in conventional diuretics (C) group (open circle) and C plus tolvaptan (T) group at the beginning of the observational period and I and 12 months after that.

Note: $* * p<0.01$.

Abbreviations: CKD, chronic kidney disease; eGFR, estimated glomerular filtration rate.

A previous study suggested that hydration may slow the progressive decline of eGFR in CKD. ${ }^{15}$ Furthermore, an extended review showed that vasopressin receptor V1-3 is a novel target to ameliorate the progression of $\mathrm{CKD} .{ }^{15}$ Because tolvaptan is a selective antagonist of the $\mathrm{V} 2$ receptor, it inhibits urinary concentrating ability. As a result, tolvaptan may counter the slowing of urinary flow and the increased urine concentration in medullary tubules, resulting in a rise of the urine-to-plasma urea ratio. In turn, this should increase GFR via the tubule-glomerular feedback. This last effect was shown in rat models to promote the decline of GFR in the progression of glomerular sclerosis. ${ }^{16}$

Other explanations are speculative from the animal models of kidney failure. Rats treated with puromycin, a model of massive proteinuria, showed amelioration of podocyte injury as well as proteinuria after application of tolvaptan. ${ }^{17}$ It is also shown in another study that tolvaptan can decrease proteinuria, ${ }^{18}$ although the amount of proteinuria did not change during the course in our study. Moreover, Ishikawa, et $\mathrm{a}^{19}$ demonstrated that a heart failure model produced in Dahl rats experienced tubulointerstitial damage, which was restored by the administration of tolvaptan. These potential benefits of tolvaptan on kidney injuries in light of our results should be investigated in future clinical trials. 
This study had several limitations. First, it was an observational study and thus cannot draw conclusion about causative relationships. Second, a limited number of patients were included in the study. Third, the $\mathrm{C}$ group was not selected in a random matter. Although we tried to remove biases by matching treatment and control patients by demographic factors, selection biases may remain. Last, the dosage of diuretics was determined by the physician in charge of the patients. However, the volume status of the patients was not different between the 2 groups.

Despite these limitations, this is the first study to demonstrate the usefulness of tolvaptan in the patients with stage 3a-4 CKD. Thus, the present study may form the basis for future randomized clinical trials that assess the efficacy of tolvaptan in slowing the decline of renal function in CKD.

\section{Conclusion}

In conclusion, tolvaptan used in addition to conventional diuretics for the control of fluid overload may be associated with the slower decline of eGFR in stage 3-4 CKD even without multiple renal cysts or heart failure.

\section{Acknowledgment}

The authors wish to acknowledge the contribution of the medical staff at the Department of Nephrology, Kawaguchi Saisekai Hospital, Saitama, Japan.

\section{Disclosure}

The authors report no conflicts of interest in this work.

\section{References}

1. Khan YH, Sarriff A, Adnan AS, Khan AH, Mallhi TH. Outcomes of diuretic use in pre-dialysis CKD patients with moderate renal deterioration attending tertiary care referral center. Clin Exp Nephrol. 2017;21(6):1011-1023.

2. Ikeda S, Ohshima K, Miyazaki S, et al. Impact of chronic kidney disease on the diuretic response of tolvaptan in acute decompensated heart failure. ESC Heart Fail. 2017;4(4):614-622.
3. Tominaga $\mathrm{N}$, Kida $\mathrm{K}$, Inomata $\mathrm{T}$, et al. Effects of tolvaptan addition to furosemide in normo- and hyponatremia patients with heart failure and chronic kidney disease stages G3b-5: a subanalysis of the K-STAR Study. Am J Nephrol. 2017;46(5):417-426.

4. Torres VE, Chapman AB, Devuyst O, et al. Tolvaptan in later-stage autosomal dominant polycystic kidney disease. $N$ Engl J Med. 2017;377(20):1930-1942.

5. Sato E, Nakamura T, Amaha M, et al. Effect of tolvaptan in patients with chronic kidney disease due to diabetic nephropathy with heart failure. Int Heart J. 2014;55(6):533-538.

6. Matsuo S, Imai E, Horio M, et al. Revised equations for estimated GFR from serum creatinine in Japan. Am J Kidney Dis. 2009;53(6):982-992.

7. Nitta K, Kawashima A, Yumura W, et al. Plasma concentration of brain natriuretic peptide as an indicator of cardiac ventricular function in patients on hemodialysis. Am J Nephrol. 1998;18(5):411-415.

8. Akamatsu M, Ogawa T, Fujiu A, Matsuda N, Nitta K. Clinical assessment of atherosclerotic parameters and cardiac function in chronic hemodialysis patients. Clin Exp Nephrol. 2009;13(6):651-658.

9. Tahara T, Mori K, Mochizuki M, et al. Tolvaptan is effective in treating patients with refractory ascites due to cirrhosis. Biomed Rep. 2017;7(6):558-562.

10. Park ES, Huh YS, Kim GH. Is tolvaptan indicated for refractory oedema in nephrotic syndrome? Nephrology (Carlton). 2015;20(2):103-106.

11. Wu MY, Chen TT, Chen YC, et al. Effects and safety of oral tolvaptan in patients with congestive heart failure: a systematic review and network meta-analysis. PLoS One. 2017;12(9):e0184380.

12. Katsumata M, Hirawa N, Sumida K, et al. Effects of tolvaptan in patients with chronic kidney disease and chronic heart failure. Clin Exp Nephrol. 2017;21(5):858-865.

13. Torres VE, Higashihara E, Devuyst O, et al. Effect of tolvaptan in autosomal dominant polycystic kidney disease by CKD stage: results from the TEMPO 3:4 trial. Clin JAm Soc Nephrol. 2016;11(5):803-811.

14. Muto S, Okada T, Yasuda M, Tsubouchi H, Nakajima K, Horie S. Long-term safety profile of tolvaptan in autosomal dominant polycystic kidney disease patients: TEMPO Extension Japan Trial. Drug Healthc Patient Saf. 2017;9:93-104.

15. Clark WF, Sontrop JM, Huang SH, Moist L, Bouby N, Bankir L. Hydration and chronic kidney disease progression: a critical review of the evidence. Am J Nephrol. 2016;43(4):281-292.

16. Bankir L, Bouby N, Ritz E. Vasopressin: a novel target for the prevention and retardation of kidney disease? Nat Rev Nephrol. 2013;9(4):223-239.

17. Ishibashi $\mathrm{K}$, Hara $\mathrm{S}$, Kondo S. Aquaporin water channels in mammals. Clin Exp Nephrol. 2009;13(2):107-117.

18. El Boustany R, Taveau C, Chollet C, et al. Antagonism of vasopressin V2 receptor improves albuminuria at the early stage of diabetic nephropathy in a mouse model of type 2 diabetes. J Diabetes Complications. 2017;31(6):929-932.

19. Ishikawa M, Kobayashi N, Sugiyama F, Onoda S, Ishimitsu T. Renoprotective effect of vasopressin V2 receptor antagonist tolvaptan in Dahl rats with end-stage heart failure. Int Heart J. 2013;54(2):98-106.
The International Journal of Nephrology and Renovascular Disease is an international, peer-reviewed open access journal focusing on the pathophysiology of the kidney and vascular supply. Epidemiology, screening, diagnosis, and treatment interventions are covered as well as basic science, biochemical and immunological studies. The manuscript

\section{Dovepress}

management system is completely online and includes a very quick and fair peer-review system, which is all easy to use. Visit http://www. dovepress.com/testimonials.php to read real quotes from published authors. 